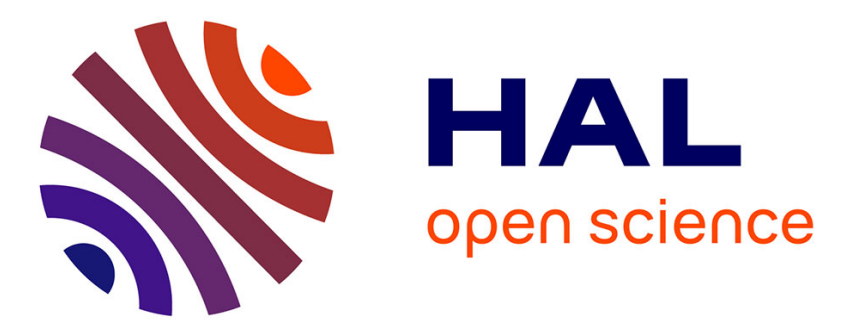

\title{
Joint QoS control for video streaming over wireless multihop networks: A cross-layer approach
} Liang Zhou, Baoyu Zheng, Benoit Geller, Anne Wei, Jingwu Cui

\section{To cite this version:}

Liang Zhou, Baoyu Zheng, Benoit Geller, Anne Wei, Jingwu Cui. Joint QoS control for video streaming over wireless multihop networks: A cross-layer approach. AEU - International Journal of Electronics and Communications, 2009, 10.1016/j.aeue.2008.05.004 . hal-01225693

\section{HAL Id: hal-01225693 \\ https://hal.science/hal-01225693}

Submitted on 4 Dec 2015

HAL is a multi-disciplinary open access archive for the deposit and dissemination of scientific research documents, whether they are published or not. The documents may come from teaching and research institutions in France or abroad, or from public or private research centers.
L'archive ouverte pluridisciplinaire HAL, est destinée au dépôt et à la diffusion de documents scientifiques de niveau recherche, publiés ou non, émanant des établissements d'enseignement et de recherche français ou étrangers, des laboratoires publics ou privés. 


\title{
Joint QoS Control for Video Streaming over
}

Wireless Multihop Networks: A Cross-Layer

\section{Approach}

\author{
Liang Zhou, Baoyu Zheng, Benoît Geller, Anne Wei, Jingwu Cui
}

\begin{abstract}
In this paper, we propose a novel cross-layer framework for jointly controlling and coding for multiple video streams in wireless multihop networks. At first, we develop a cross-layer flow control algorithm which works at the medium access control (MAC) layer to adjust each link's persistence probability and at the transport layer to adjust flow rates. This proposal is designed in distributed manner that is amenable to on-line implementation for wireless networks. And then, a rate-distortion optimized joint source-channel coding (JSCC) approach for error-resilient scalable encoded video is presented, in which the video is encoded into multiple independent streams and each stream is assigned forward error correction (FEC) codes to avoid error propagation. Furthermore, we integrate the JSCC with the specific flow control algorithm, which optimally applies the appropriate channel coding rate given the constraints imposed by the transmission rate obtained from the proposed flow control algorithm and the prevailing channel condition. Simulation results demonstrate the merits and the need for joint QoS control in order to provide an efficient solution for video streaming over wireless multihop networks.
\end{abstract}

\section{Index Terms}

video, QoS, wireless networks, cross-layer, flow control

Corresponding Author: Liang Zhou (Email: zkingzll1981@gmail.com)

L. Zhou, B. Zheng and J. Cui are with the Electric Engineering Department, Shanghai Jiao Tong University, Shanghai, China and with the Institute of Signal Processing and Transmission, Nanjing University of Posts and Telecommunications, Nanjing, China; B. Geller is with the SATIE, Ecole Normale Supérieure (E.N.S.) Cachan and with the LEI, Ecole Nationale Supérieure des Techniques Avancées, Paris, France; A. Wei is with the CEDRIC, Conservation National des Arts et Métiers, Paris, France. 


\section{INTRODUCTION}

Rapid growth in wireless networks is fueling the demand that services traditionally available only in wired networks, such as video, be available to mobile users. However, the characteristics of wireless systems provide a major challenge for reliable transport of video since the video transmitted over wireless channels is highly sensitive to noise, interference and topology change which can cause both packet losses and bit-errors. Furthermore, these errors tend to occur in bursts, which can further decrease the delivered Quality of Service (QoS). Current and future wireless systems will have to cope with this lack of QoS guarantees.

To date, the video transmission over wireless networks is still an open problem. [4], [5] and [8] present some wavelet-based encoders that divide the original bit-stream into multiple streams, called multiple description coding (MDC), to tradeoff the error-resilience and coding complexity; [6] and [7] amplify the benefits of using MDC by combining with path diversity, in these approaches each stream is explicitly transmitted over an independent path to the receiver to achieve higher tolerance to packet loss and delay due to network congestion; In [3], the authors propose two flow control algorithms for network with multiple paths between source-destination pair, both are distributed algorithms over the network to maximize aggregate source utility. However, these works, as well as much previous work appearing in the literature, target the problem from an individual point of view without considering the overall system performance. Therefore, it remains unclear what level of video quality can be supported by the wireless networks.

Typically, for video communications over wireless multihop networks, there are two main factors which can greatly affect the perceived video quality: the transmission error and flow control. On one hand, packet loss leading to transmission error is generally caused by channel fading, multi-path effects, interference from other electronic devices, and node mobility. In addition, the video coding standards, including MPEG-4 and H.264, are designed to achieve high compression efficiency at the expense of the error resilience. The coding efficiency in these standards is achieved by using motion-compensated prediction to reduce the temporal and statistical redundancy between the video frames. This poses a severe problem, namely error propagation, where errors due to packet loss in a reference frame propagate to all of the dependent frames leading to visual artifacts that can be long lasting and annoying [1]. On the other hand, the 
optimal flow control is the most reasonable operating rate that can be reliably supported along a path. If the operating rate is lower than the optimal transmission rate along a path, performance loss will occur due to the source coding inefficiency. On the contrary, if the operating rate is higher, many packets will be discarded due to channel over-pumping [15]. Thus, a flow control scheme is both desirable and necessary to achieve a satisfactory level of received video quality over wireless networks [16]. In addition, the flow control scheme must be designed in distributed manner that is amenable to online implementation for wireless network [2]. However, to the best of our knowledge, most of the current literatures has considered above two factors separately and independently. In order to achieve improved video quality supported by wireless networks, and to provide a more robust video delivery system, these two factors are jointly considered in this paper.

The rest of paper is organized as follows. In Section II, we propose a cross-layer distributed flow control algorithm. In Section III, an analytical rate-distortion optimized JSCC is proposed given the optimal transmission rate. And then, we present some selected simulation results for the proposed joint scheme transmission over wireless networks in Section IV, followed by concluding remarks in Section V.

\section{Distributed Flow Control Algorithm}

\section{A. Network Model}

Consider this wireless multihop network whose links are denoted by $\boldsymbol{L}=\{1 \ldots, l, \ldots L\}$. Let $c_{l}$ be the capacity of link $l \in \boldsymbol{L}$ and $c=\left[c_{1} \ldots, c_{l}, \ldots, c_{L}\right]^{T}$. Let $\boldsymbol{S}=\{1 \ldots, s, \ldots S\}$ be the set of sources. Each source $s \in \boldsymbol{S}$ has $n_{s}$ potential available paths from the source to the destination. Let the $L \times 1$ vector $\boldsymbol{R}_{s, i}$ denotes the set of links used by source $s \in \boldsymbol{S}$ on its path $i \in\left\{1,2, \ldots, n_{s}\right\}$, whose $l$ th element equals to 1 if path contains link $l$ and 0 otherwise, and $N_{s, i}$ denotes the number of links on the path $i$. The set of all the available paths of user $s$ is defined by

$$
\boldsymbol{R}_{s}=\left[\boldsymbol{R}_{s, 1}, \boldsymbol{R}_{s, 2}, \ldots, \boldsymbol{R}_{s, n_{s}}\right]
$$

and the total paths in the network are defined by a routing matrix $\boldsymbol{R}$,

$$
\boldsymbol{R}=\left[\boldsymbol{R}_{1}, \ldots, \boldsymbol{R}_{s}, \ldots, \boldsymbol{R}_{S}\right]
$$

For each source $s$, let $x_{s, i}$ be the rate of source $s$ on path $\boldsymbol{R}_{s, i}$, and $x_{s}=\sum_{i=1}^{n_{s}} x_{s, i}$ be the total source rate. When each source $s$ transmits at a total rate of $x_{s}$, it attains a utility $U_{s}\left(x_{s}\right)$. Let 
$x=\left[x_{1,1}, \ldots, x_{n, n_{S}}\right]^{T}$ be the vector of all path rates of all sources. Our objective is to choose appropriate rates $x$ so as to maximize the total utility $\sum_{s \in S} U_{s}\left(x_{s}\right)$ :

$$
\begin{array}{rc}
\max _{x_{s} \geq 0} & \sum_{s \in S} U_{s}\left(x_{s}\right) \\
\text { subject to } & x_{s}=\sum_{i=1}^{n_{s}} x_{s, i}, \\
& \boldsymbol{R} x \leq c, x_{s, i} \geq 0
\end{array}
$$

In addition, each link has a contention resolution protocol based on the transmission persistence probability. Let $L_{o}(s)$ denotes the set of outgoing links from node $s \in \boldsymbol{S}$, and $N_{I}(l)$ as the set of nodes whose transmissions cause interference to the receiver of link $l$, excluding the transmitter node of link, and $L_{I}(s)$ as the set of links whose transmissions get interfered from the transmission of node $s$, excluding the outgoing links from node $s$. Therefore, if the link $l$ and a node in $N_{I}(l)$ transmit data simultaneously, the transmission of link $l$ fails. If node $s$ and a link $l$ in $L_{I}(s)$ transmit data simultaneously, the transmission of link $l$ also fails. Assuming each node $s$ transmits data with a probability $p_{s}^{\prime}$, when it determines to transit data, it chooses one of its outgoing paths with a probability $q_{l}, l \in L_{o}(s)$. Consequently, link $l \in L_{o}(s)$ transmits data with a probability $p_{l}=p_{s}^{\prime} q_{l}$, which is called persistence probability of link $l$. Therefore, the data rate on path $i, x_{s, i}$, is obtained as minimum rate of link in the path:

$$
x_{s, i}\left(P_{s}\right)=\min _{l \in\left[1, N_{s, i]}\right]} c_{l} p_{l} \prod_{k \in N_{I}(l)}\left(1-\sum_{m \in L_{o}(k)} p_{m}\right)
$$

where $P_{s}=\left[p_{1}, \ldots, p_{N_{s, i}}\right]$.

Based on this, we can specify this wireless networks utility maximization with "contention" link:

$$
\begin{array}{rc}
\max _{x_{s} \geq 0} & \sum_{s \in S} U_{s}\left(x_{s}\right) \\
\text { subject to } & x_{s}=\sum_{i=1}^{n_{s}} x_{s, i}\left(P_{s}\right) \\
& \boldsymbol{R} x \leq c, x_{s, i} \geq 0,0 \preceq P_{s} \preceq 1
\end{array}
$$

\section{B. Cross-Layer Optimal Control}

In the case of multi-path flow control problem, many literatures have been proposed by introducing the variable of path price to maximum the total utility function of the system [9], 
[10]. These works suggest ways to adapt the total source rate to congestion, but they don't specify how to distribute the total rate among the available paths. A naive approach is to simply split it evenly along paths that have the least current price. This algorithm, however, does not converge, e.g. when multiple paths have different path prices. Motivated by this approach, we can view the persistence probability as a special path price, the distributed optimal $x_{s}$ at each source can be adjusted according to the persistence probability of each link. Similarly, the persistence probability can also be adjusted according to current $x_{s}$. Intuitively, the expected result will allocate the "right" amount persistence probability to the "right" links to alleviate the congestion, which may then induce an increase in end-to-end throughput of the whole system. Therefore, we need to find an algorithm that distributively and adaptively control the flow rate, persistence probability and routing from the link and source aspects, respectively. Aiming for this, we propose a cross-layer optimal control (CLOC) algorithm as follows:

we assume that time is slotted, and the links are fixed within a time slot but independently change between different slots. During each time slot $t$, the following three units are carried out simultaneously.

- Step 1: Each link $l$ receives flow rates $x_{s, i}(t)$ for all paths $\boldsymbol{R}_{s, i}$ that contain link $l$, and computes a new persistence probability

$$
p_{l}(t+1)=\left[p_{l}(t)+\kappa \frac{c_{l}-\sum_{s} \boldsymbol{R}_{s, i} x}{p_{l}(t)}-\kappa \frac{\sum_{k \in L_{I}\left(t_{l}\right)} \varepsilon_{k}}{1-\sum_{m \in L_{o}\left(t_{l}\right)} p_{m}}\right]_{0}^{1}
$$

where the second term is the residual link capacity relative to current persistence probability and the third term is the interference penalty. $t_{l}$ is the transmitter node whose path contains link $l$, $\kappa>0$, and

$$
\varepsilon_{l}=\left\{\begin{array}{c}
0, \text { if } \sum_{s} x_{s, i}(t) \leq c_{l} p_{l} \prod_{k \in N_{I}(l)}\left(1-\sum_{m \in L_{o}(k)} p_{m}\right) \\
1, \quad \text { otherwise }
\end{array}\right.
$$

Informing new probability $p_{l}(t+1)$ to all sources $s$ whose path $\boldsymbol{R}_{s, i}$ contains link $l$; and each source receives $p_{s, i}^{r}=P_{s}^{T} \boldsymbol{R}_{s, i}$ from the network for all its paths $\boldsymbol{R}_{s, i}, i=1,2, \ldots, n_{s}$ and decides the maximum persistence probability $p_{s}^{r^{*}}=\max _{i=1,2, \ldots, n_{s}} p_{s, i}^{r}(t)$.

- Step 2: Updates the source rate $x_{s}(t+1)$ :

$$
x_{s}(t+1)=\left[U_{s}^{\prime-1}\left(p_{s}^{r^{*}}(t)\right)\right]_{m_{s}}^{M_{s}}
$$


To these paths which do not have the maximum persistence probability, path rate $x_{s, i}(t+1)$ on path $\boldsymbol{R}_{s, i}$ is:

$$
x_{s, i}(t+1)=\left[x_{s, i}(t)-\gamma\left(p_{s}^{r^{*}}(t)-p_{s, i}^{r}(t)\right)\right]^{+}, \gamma>0
$$

To the path $\boldsymbol{R}_{s, j}$ that has the maximum persistence probability, we set its rate as:

$$
x_{s, j}(t+1)=\left[x_{s}(t+1)-\sum_{i \in\left[1, n_{s}\right], i \neq j} x_{s, i}(t+1)\right]^{+}
$$

and then informs all the new flow rate $x_{s, j}(t+1)$ to links $l$ contained in path $\boldsymbol{R}_{s, i}$.

- Step 3: Over the chosen link, send an amount of bits to destination according to the rate determined by the rate control unit.

\section{Convergence Theorem of CLOC}

We first associate Lagrange multiplier for each of the constraints, and use the KKT optimality conditions for optimization [11], solving this problem is equivalent to satisfying the complementary slackness condition and finding the stationary points of the Lagrange.

$$
\begin{aligned}
L_{s y s} & =L\left(x, P_{s}, \bar{\lambda}, \underline{\lambda}\right) \\
& =\sum_{s \in S} U_{s}\left(x_{s}\right)-\bar{\lambda}_{s}\left(x_{s}-\sum_{i=1}^{n_{s}} x_{s, i}\left(P_{s}\right)\right)-\underline{\lambda}_{l}^{T}(\boldsymbol{R} x-c) \\
& =\left(\sum_{s \in S} U_{s}\left(x_{s}\right)-\bar{\lambda}_{s} x_{s}\right)+\bar{\lambda}_{s} \sum_{i=1}^{n_{s}} x_{s, i}\left(P_{s}\right)-\underline{\lambda}_{l}^{T}(\boldsymbol{R} x-c)
\end{aligned}
$$

where $\bar{\lambda}=\left[\bar{\lambda}_{1}, \bar{\lambda}_{2}, \ldots, \bar{\lambda}_{S}\right], \underline{\lambda}=\left[\underline{\lambda}_{1}, \underline{\lambda}_{2}, \ldots, \underline{\lambda}_{L}\right]^{T}$. By linearity of the differentiation operator, this can be decomposed into two separate maximization problems

$$
\begin{aligned}
& \max _{x \succeq 0} L_{x}(x, \bar{\lambda})=\sum_{s \in S} U_{s}\left(x_{s}\right)-\bar{\lambda}_{s} x_{s} \\
& \max _{P_{s} \succeq 0} L_{P_{s}}\left(\bar{\lambda}, \underline{\lambda}, P_{s}\right)=\bar{\lambda}_{s} \sum_{i=1}^{n_{s}} x_{s, i}\left(P_{s}\right)-\underline{\lambda}_{l}^{T}(\boldsymbol{R} x-c) \\
&=\bar{\lambda}_{s} \sum_{i=1}^{n_{s}}\left(\min _{l \in\left[1, N_{s, i}\right]} c_{l} p_{l} \prod_{k \in N_{I}(l)}\left(1-\sum_{m \in L_{o}(k)} p_{m}\right)\right)-\underline{\lambda}_{l}^{T} p_{l}\left(x_{l}-c_{l}\right)
\end{aligned}
$$

The first maximization is already implicitly solved by the congestion control mechanism for different $U_{s}$ [13], while the second maximization also need to solve which aims at allocating 
exactly the right persistence probability of each link to reduce the congestion at the network bottlenecks. Here, we focus on proving the convergence of the persistence probability problem.

Because max $L_{P_{s}}\left(P_{s}\right)$ is a typical non-concave/convex problem, it is difficult to take the derivative of $L_{P_{s}}\left(P_{s}\right)$ with respect to $p_{l}$ directly. To get around the difficulty, we take logarithm operator on $L_{P_{s}}\left(P_{s}\right)$ to get $L_{P}(\widetilde{P})$, that is $L_{P_{s}}\left(\widetilde{P_{s}}\right)=\min _{l \in\left[1, N_{s, i}\right]} \sum_{i=1}^{n_{s}} \log \left(c_{l} p_{l} \prod_{k \in N_{I}(l)}\left(1-\sum_{m \in L_{o}(k)} p_{m}\right)\right)+$ $\log p_{l}\left(x_{l}-c_{l}\right)$, where $\tilde{p}_{l}=\log p_{l}$. In Appendix I, we will show that the partial Lagrange is a strictly concave function of a logarithmically transformed probability vector. So we can decompose that

$$
\begin{aligned}
L_{P_{s}}\left(\widetilde{P_{s}}\right) & =\min _{l \in\left[1, N_{s, i}\right]} \sum_{i=1}^{n_{s}} \log \left(c_{l} p_{l} \prod_{k \in N_{I}(l)}\left(1-\sum_{m \in L_{o}(k)} p_{m}\right)\right)+\log p_{l}\left(x_{l}-c_{l}\right) \\
& =\sum_{i=1}^{n_{s}} \log \left[c_{l} p_{l} \prod_{k \in N_{I}(l)}\left(1-\sum_{m \in L_{o}(k)} p_{m}\right)-\sum_{n \in L_{I}\left(t_{l}\right)} c_{n} p_{n} \prod_{k \in N_{t o}^{I}(n), k \neq t_{l}}\left(1-\sum_{m \in L_{o}(k)} p_{m}\right)\right] \\
& +\log p_{l}\left(c_{l}-\sum_{s} \boldsymbol{R}_{s, i} x\right)
\end{aligned}
$$

Assuming $\varepsilon_{l}$ as (7), taking the derivative of $L_{P_{s}}\left(\tilde{P}_{s}\right)$ with respect to $\tilde{P}_{s}$, we have

$$
\begin{aligned}
\nabla_{l} L_{P_{s}}\left(\tilde{P}_{s}\right) & =\left(c_{l}-\sum_{s} \boldsymbol{R}_{s, i} x\right)-p_{l} \frac{\prod_{k \in N_{I}(l)}\left(1-\sum_{m \in L_{o}(k)} p_{m}\right) \varepsilon_{k}}{1-\sum_{m \in L_{o}\left(t_{l}\right)} p_{m}} \\
& =\left(c_{l}-\sum_{s} \boldsymbol{R}_{s, i} x\right)-p_{l} \frac{\sum_{k \in L_{I}\left(t_{l}\right)} \varepsilon_{k}}{1-\sum_{m \in L_{o}\left(t_{l}\right)} p_{m}}
\end{aligned}
$$

Coming back to the $P_{s}$ solution space instead of $\tilde{p}_{l}$, it is easy to verify that the derivate of $L_{P_{s}}\left(P_{s}\right)$ with respect to $p_{l}$ is

$$
\nabla_{l} L_{P_{s}}\left(P_{s}\right)=\frac{c_{l}-\sum_{s} \boldsymbol{R}_{s, i} x}{p_{l}}-\frac{\sum_{k \in L_{I}\left(t_{l}\right)} \varepsilon_{k}}{1-\sum_{m \in L_{o}\left(t_{l}\right)} p_{m}}
$$

Therefore, the logarithmic change of variables simply scales each entry of the gradient by $p_{l}$ : $\nabla_{l} L_{P_{s}}\left(P_{s}\right)=\nabla_{l} L_{P_{s}}\left(\tilde{P}_{s}\right) / p_{l}$. We now use the subgradient method [14], with a constant step size $\kappa$, to maximize $L_{P_{s}}\left(P_{s}\right)$

$$
\begin{aligned}
p_{l}(t+1) & =p_{l}(t)+\kappa \nabla_{l} L_{P_{s}}\left(P_{s}\right) \\
& =\left[p_{l}(t)+\kappa \frac{c_{l}-\sum_{s} \boldsymbol{R}_{s, i} x}{p_{l}(t)}-\kappa \frac{\sum_{k \in L_{I}\left(t_{l}\right)} \varepsilon_{k}}{1-\sum_{m \in L_{o}\left(t_{l}\right)} p_{m}}\right]_{0}^{1}
\end{aligned}
$$




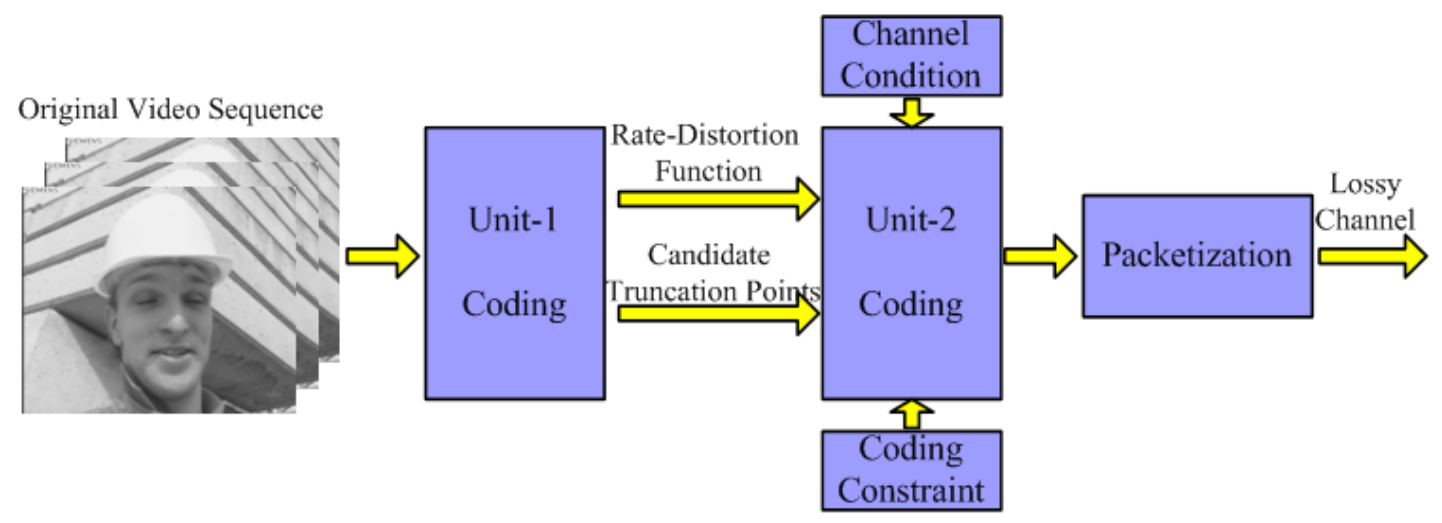

Fig. 1. The architecture of JSCC.

This is the exact Step 1 in the CLOC algorithm. Substituting the known equation into $x_{s}(t+1)=$ $\left[U_{s}^{\prime-1}\left(p_{s}^{r^{*}}(t)\right)\right]_{m_{s}}^{M_{s}}$ to compute the total rate of $s$ at $t+1$, the following is the same as the proof in [9]. Since $x_{s, i}$ can be turned into a concave function in $P_{s}$, each constraint $x_{s} \leq \sum_{i=1}^{n_{s}} x_{s, i}\left(P_{s}\right)$ is upper bound constraint on a convex function in $\left(x, P_{s}\right)$, problem (5) can be turned into maximizing a strictly concave objective function over a convex constraint set [12]. Therefore, the established convergence is towards the global optimum. It should be noted that when path number is small, few rounds of the CLOC algorithm is needed, hence lower delay for rate control and routing acquisition; when path number is large, however, the distributed algorithm may calculate too many possibilities for each potential paths, and the total video transmission may be further restricted by the long delay in each path. In order to resolve this problem, we choose the first three paths and re-allocate the total probability among them in practical implementation.

\section{JoInt SOURCE-ChANNEl CODING}

\section{A. The Architecture of JSCC}

The proposed coding architecture contains two parts as shown in Fig. 1. Unit-1 uses the 3-D SPIHT (3-D Set Partitioning In Hierarchical Trees) codec that generates independent embedded streams, while Unit-2 uses the coding constraints and channel condition to pack the bit-streams into pack-streams of quality layers. This two-units structure collects incremental contributions from the various streams into SNR scalable quality layers in a way similar to that of embedded 
block coding with optimized truncation. The streams and rate-distortion functions generated by Unit-1 can be processed independently to channel conditions. The source and channel allocation algorithm in Unit-2 must be efficient to cope with the time varying channel conditions.

Unit-1 uses an embedded coding technique that generates multiple independent embedded streams. A video is divided into several independently encoded for additional functionality in Unit-1. The video coder divides the 3-D wavelet coefficients into multiple blocks according to their spatial and temporal relationships, and then encodes each block independently using the 3D SPIHT algorithm [4]. The proposed method allocates source bits to each embedded bitstream to minimize the total distortion of a video clip. Moreover, the video scalability is imparted by the layering concept and the scalable stream is organized into quality layers.

\section{B. Packet-Loss Pattern Approximation}

For the RS code operating on $b$-bit symbols, the maximum block length is $2^{b}-1$ symbols. For a $(n, k)$ systematic RS code with a block length $n$, the source symbol is $k$. The first $k$ encoded symbols are source symbols correctly when the number of loss symbols is less than the minimum distance $d_{\text {min }}=n-k+1$ of the code. The performance of an RS decoder $P_{c}(n, k)$ can be characterized by the code correct probability

$$
P_{c}(n, k)=\sum_{m=0}^{n-k} P(n, m)
$$

where $P(n, m)$ is the probability of $m$ erasure within a block of $n$ symbols. In a binary symmetric channel without memory, we have

$$
P(n, m)=\left(\begin{array}{c}
n \\
m
\end{array}\right) P_{B}^{m}\left(1-P_{B}\right)^{n-m}
$$

where $P_{B}$ is the mean packet loss rate. For channels with memory, it is more complicated to calculate the $P(n, m)$. Here, we use a two-state Markov model (i.e.Gilbert model) to simulate the bursty packet loss behavior. The two states of this model are denoted as G (Good) and B (Bad). In state $\mathrm{G}$, packets are received correctly and timely, whereas, in state B, packets are assumed to be lost. This model can be described by the transition probabilities $P_{G B}$ from state $\mathrm{G}$ to $\mathrm{B}$ and $P_{B G}$ from state $\mathrm{B}$ to $\mathrm{G}$. The then the average error probability $P_{B}$ is given by

$$
P_{B}=\frac{P_{G B}}{P_{G B}+P_{B G}}
$$


and the average burst length

$$
L_{B}=\frac{1}{P_{B G}}
$$

which is the average number of consecutive symbol errors. The Markov model is a renewal model, and such models are determined by the distribution of error-free intervals, known as gap. Let gap of length $\sigma$ be the event that after a lost packet, $\sigma-1$ packets are received correctly and then a packet is lost again. The gap density function $g(\sigma)$ gives the probability of a gap length $\sigma$. The gap distribution function $G(\sigma)$ gives the probability of the gap length greater than $\sigma-1$. These functions can be derived as [5]

$$
\begin{gathered}
g(\sigma)=\left\{\begin{array}{cc}
1-P_{B G}, & \sigma=1 \\
P_{B G}\left(1-P_{G B}\right)^{\sigma-2} P_{G B}, & \sigma>1
\end{array}\right. \\
G(\sigma)=\left\{\begin{array}{cc}
1-P_{B G}, & \sigma=1 \\
P_{B G}\left(1-P_{G B}\right)^{\sigma-2}, & \sigma>1
\end{array}\right.
\end{gathered}
$$

Let $R(n, m)$ be the probability of $m-1$ erroneous symbols within the next $n-1$ symbols following an erroneous symbol. It can be calculated using the recurrence

$$
R(n, m)=\left\{\begin{array}{cc}
G(n), & m=1 \\
\sum_{\sigma=1}^{n-m+1} g(\sigma) R(n-\sigma, m-1), & 2 \leq m \leq n
\end{array}\right.
$$

Then the probability of errors within $m$ a block of $n$ symbols is

$$
P(n, m)=\left\{\begin{array}{cc}
\sum_{\sigma=1}^{n-m+1} P_{B} G(\sigma) R(n-\sigma+1, m), & 1 \leq m \leq n \\
1-\sum_{m=1}^{n} P(n, m), & m=0
\end{array}\right.
$$

\section{Proposed JSCC}

Fig. 2 shows how multiple encoded sequences of different quality levels are protected based on systematic RS codes. For notational convenience, we define the bit-plane 1 as the highest bit-plane and the bit-plane $I_{s}$ as the lowest bit-plane to be sent for stream-s. As to the definition of the bit-plane, we employ the priority classification used in [5]. Let $N_{s}$ be the number of packets that are used to send the combined source data and redundancy for stream-s in a GOP (Group of Picture) and $L$ be the packet size in bytes. In this scheme, the bits belonging to bit-plane $i\left(1 \leq i \leq I_{s}\right)$ are $k_{s, i}$ packets and the remaining $c_{s, i}=N_{s}-k_{s, i}$ packets are filled 


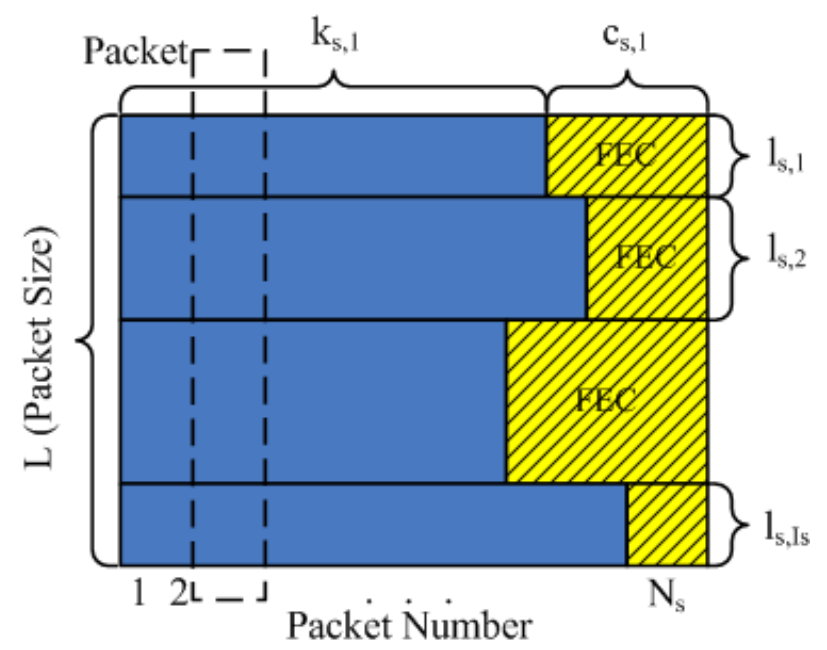

Fig. 2. Packetization of source-channel bits of different bit-layers for stream $s$.

with channel coding redundancy. In other words, the source data for bit-plane is protected by RS code $\left(N_{s}, k_{s, i}\right)$.

We denote the proposed coding scheme is performed on GOP basis. We define the total number of packets at period $t$ to be sent from all of the sources for a GOP period $N(t)$ as

$$
N(t)=\left\lceil\frac{x(t) \times N_{G O P}}{F \times L}\right\rceil
$$

where $x(t)$ is the total coding rate in bytes/s at period $t$ for the combination of data and redundancy for all sources; $N_{G O P}$ is the number of frames in one GOP; $F$ is the frame rate in frames/s. In this framework, we assume that source-s transmits stream-s to the receiver for $s \in \boldsymbol{S}$. Then the proposed algorithm divides $\left.N_{(} t\right)$ into $\left\{N_{1}(t) \ldots, N_{s}(t), \ldots N_{S}(t)\right\}$ so as to maximize the expected quality at the receiver, where $N_{s}(t)$ represents the total number of packets transmitted by source-s at GOP period $t$ for $s \in \boldsymbol{S}$. Taking account into the rate of source $s, N_{s}(t)$ should satisfy the following condition:

$$
N_{s}(t) \leq\left\lceil\frac{x_{s}(t) \times N_{G O P}}{F \times L}\right\rceil
$$

where $x_{s}(t)$ is the total rate of source-s at the GOP period $t$, and it can be obtained from the CLOC algorithm introduced in Section II-B.

Now, we can state our source and channel allocation algorithm as follows: Given $x_{s}(t)$, the proposed algorithm finds $N_{s}(t)$ and $K_{s}(t)=\left(k_{s, 1}(t), k_{s, 2}(t), \ldots, k_{s, I_{s}}(t)\right)$ for $s \in \boldsymbol{S}$, that maximize 
the expected quality at the receiver given by

$$
\begin{gathered}
P S N R(t)=\sum_{s \in S} \sum_{l=1}^{I_{s}}\left(\sum_{j=N_{s}-k_{s, l}+1}^{N_{s}-k_{s, l-1}} P\left(N_{s}(t), j\right) \sum_{i=l}^{I_{s}} P S N R_{s}(i)\right) \\
\text { subject to } \quad \sum_{s \in S} N_{s}(t)=N(t), \\
N_{s}(t) \leq\left\lceil\frac{x_{s}(t) \times N_{G O P}}{F \times L}\right\rceil
\end{gathered}
$$

$P\left(N_{s}(t), j\right)$ is the probability that $j$ packets are lost out of $N_{s}(t)$ packets sent by source-s; $P S N R_{s}(i)$ is the expected quality at the receiver when the receiver decodes up to the $i$ th bitplane for stream-s; $I_{s}$ is the last bit-plane sent from source-s.

Each source independently runs the proposed flow rate algorithm to get its optimal number of packets to transmit for a GOP period, using the information contained in the control packets that the receiver sends to all sources. The proposed algorithm tries all possible combinations of $\left(N_{s}, K_{s}\right)$ that satisfy the constraints in (28) and choose one that maximizes the expected quality. In this work, we have implemented a simple algorithm for finding the global optimal value through exhaustive search. In our future work, we will explore more efficient optimization algorithm to achieve lower complexity.

\section{Simulation Results and Discussion}

\section{A. Experimental Setup}

In order to provide a representative evaluation of system performance, for each simulation run we generate a random topology in the disc of $500 \mathrm{~m}$-by-500m square as a $2 \mathrm{D}$ Poisson point process with total number of nodes equal to 15 . Nodes within $150 \mathrm{~m}$ of each other are considered neighbors, and can communicate directly. We assume that if the distance between the transmitter node and the receiver node is less than $50 \mathrm{~m}$, the receiver node gets interference from the transmitter node. For the link from node $\mathrm{i}$ to node $\mathbf{j}\left(l_{i j}\right)$, its capacity $c_{l_{i j}}$ is computed as:

$$
c_{l_{i j}}=\frac{B W}{2} \log \left(1+\zeta S I N R_{i j}\right)
$$

where signal-to-interference-plus-noise-ratio $S I N R_{i j}$ is calculated assuming simultaneous fixed power transmission at all nodes, $B W$ is the double-sided bandwidth for transmission, and 


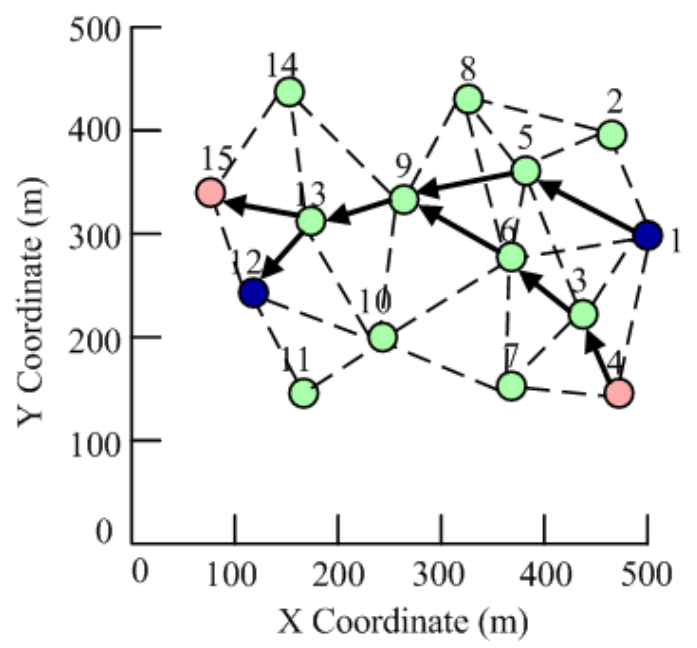

Fig. 3. An example for wireless multihop network with 15 nodes randomly placed in a 500m-by-500m area. Lines indicates the link between two neighboring nodes. Video traffics are streamed from node 1 to node 12 and node 4 to node 15 .

the coding gain $\zeta<1$ indicates the performance gap of RS coder with respect to Shannons information theoretical limit.

In the following simulations, we set $U_{s}\left(x_{s}\right)=\log _{10} x_{s}$, step-size $\kappa=\gamma=0.1$. Standard video sequence, Weather Forecast, is used at $F=30 \mathrm{fps}, N_{G O P}=30$. We use 3 levels of spatial wavelet decomposition with 9-7 biorthogonal filters and 3 levels of temporal wavelet decomposition with a Haar filter. The number of the bit-plane $I_{s}$ for each source is 3 for all experiments. For simplicity, we use the constant bit rate (CBR) model to represent video traffic. In the following, assuming two streams transmit data simultaneously from the two sources to two receivers. Fig. 3 shows the topology and routing for sources (node 1 and node 4) simultaneously stream to receivers (node 12 and node 15) over different paths. Fig. 4 shows the convergence of link persistence probabilities regulated by CLOC for the potential links from node 1 (Note: the initial persistence probability of each link is random distributed from 0 to 1 ).

Given a randomly generated topology, we initially choose a source-destination pair and stream the video from the source to the destination using the path with the highest transmission rate as described in Section II. During transmission, the environment are updated every one GOP transmission which can cause changes in the transmission rate and channel conditions. During successive one GOP transmission interval, the environment keeps constant. In order for objective 


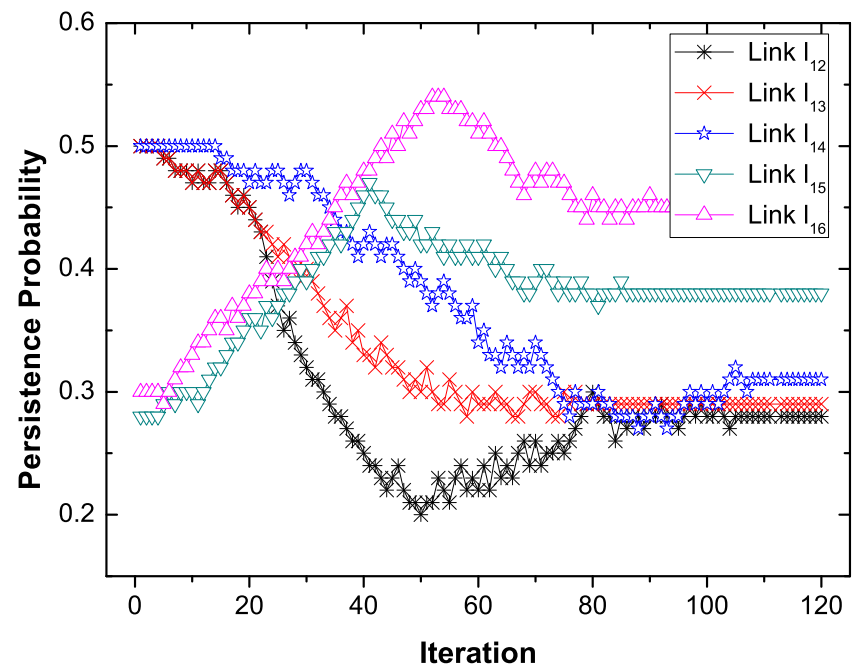

Fig. 4. Convergence of link persistence probabilities for node 1, corresponding to the same setting in Fig. 3.

TABLE I

Corresponding Channel Profile to Fig. 5(KBPS)

\begin{tabular}{|c|c|c|c|c|}
\hline GOP No. & 1 & 2 & 3 & 4 \\
\hline$x_{1}$ & 105 & 196 & 77 & 122 \\
\hline$R_{f}$ & 96 & 96 & 96 & 96 \\
\hline
\end{tabular}

comparison, PSNR (Peak Signal-to-Noise Ratio) at the receiver relative to the original video sequence is used and its definition is

$$
\operatorname{PSNR}(d B)=10 \log _{10}\left(\frac{255^{2}}{M S E}\right)
$$

where MSE is the mean-square error between the original and the decoded luminance frame.

\section{B. Selected Simulation Results and Discussions}

To demonstrate the effectiveness of our proposed rate control algorithm, we use a representative drop-tail scheme for comparison which does not use rate control. More specifically, it employs a 


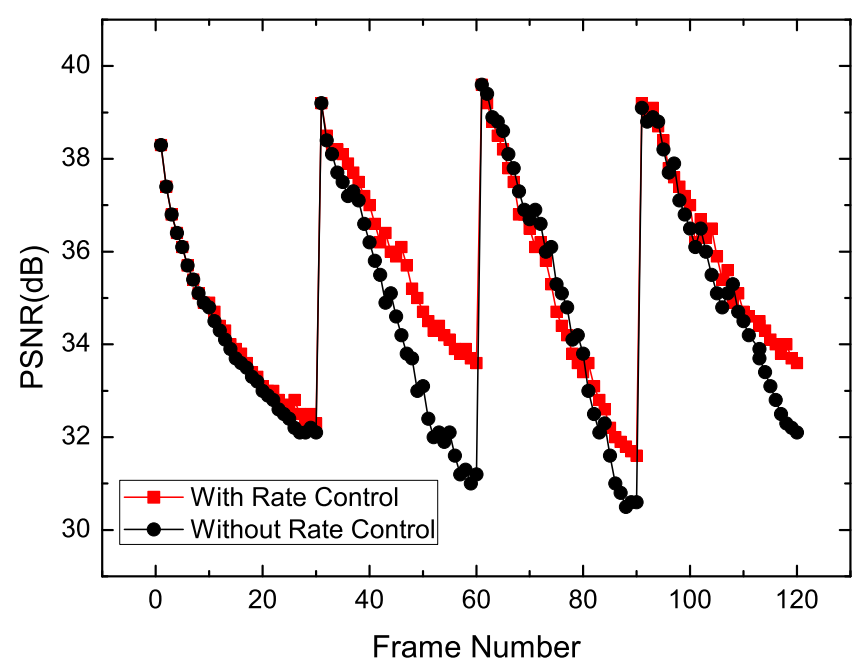

Fig. 5. Performance comparison between using rate control and without rate control (fixed source coding 96Kbps)

TABLE II

Corresponding Channel Profile to Fig. 6

\begin{tabular}{|c|c|c|c|c|}
\hline GOP No. & 1 & 2 & 3 & 4 \\
\hline$x_{4}(\mathrm{Kbps})$ & 110 & 144 & 205 & 196 \\
\hline$P_{B}(\%)$ & 5.3 & 9.6 & 8.4 & 3.2 \\
\hline$L_{B}$ & 4.6 & 5.4 & 4.4 & 3.8 \\
\hline
\end{tabular}

fixed source coding rate $R_{f}=96 \mathrm{Kbps}$ and when the rate exceeds the current optimal transmission rate available for the selected source-destination pair, it will drop the subsequent encoded packets. In Fig. 5, we show a performance comparison between our proposed rate control scheme and the drop-tail scheme in the scenario where packet losses are caused only by channel over-pumping ${ }^{1}$. It should be noted that due to the use of CBR encoding, the video quality is not constant and varies periodically. In Fig. 5, the average PSNR using the proposed rate control scheme is 35.33 $\mathrm{dB}$ while it is $34.70 \mathrm{~dB}$ for the case of no rate control, thus, $0.63 \mathrm{~dB}$ performance gain can be

\footnotetext{
${ }^{1}$ Here, we assume that no transmission errors occurred
} 


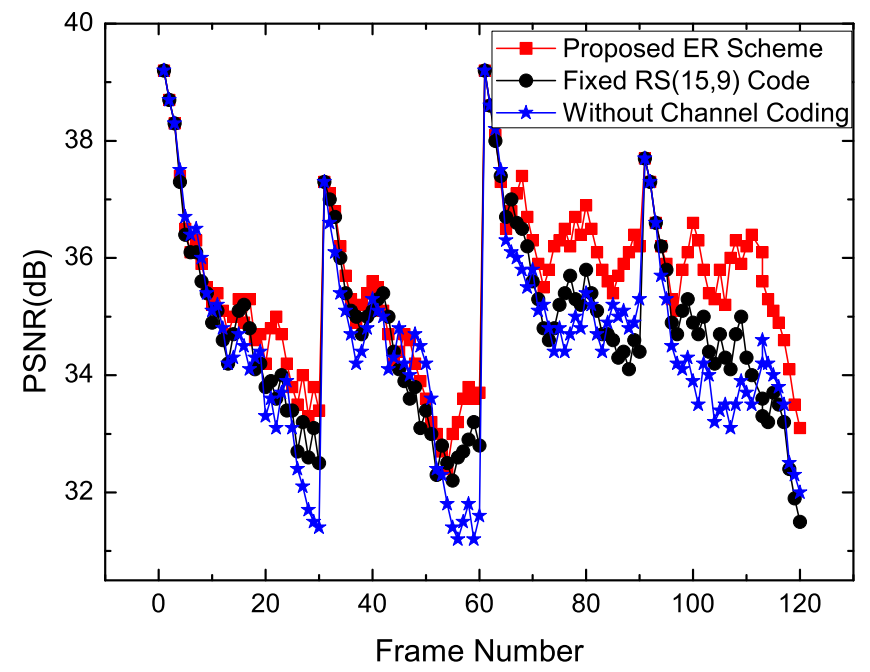

Fig. 6. Performance comparison between the proposed JSCC scheme with the representative fixed channel coding and without channel coding scheme.

achieved using the proposed rate control scheme. From the channel profile, illustrated in Table I, we can see that for GOP No. 1, No. 2, and No. 4, the optimal transmission rate is higher than the fixed 96 Kbps. Thus, using rate control can fully exploit the optimal transmission rate resulting in improved performance compared to using a fixed-rate coding scheme. On the other hand, for GOP No. 3, it is obvious that the fixed source coding rate is higher than the optimal transmission rate. Therefore, packet losses will occur when the transmission buffer is full resulting in the last couple of frames being lost which cause substantial performance degradation. A lost frame is concealed by just copying the previous frame and if several consecutive frames are lost, the degradation will be even more serious since the concealed frames are then used as correctly received frames to conceal the subsequent lost frames. This results in substantial error propagation. For example, in Fig. 5, we can see that there is substantial performance degradation around the 90th frame for the no-rate control case due to channel over-pumping. Furthermore, although the performance degradation caused by the channel over-pumping packet losses has been partially compensated using passive error concealment, the performance is still not as good as using the rate-control scheme. 
TABLE III

PERFORMANCE COMPARISON FOR OTHER SEQUENCE UNDER DIFFERENT SIMULATION CONDITIONS

\begin{tabular}{c|c|c|c|c|c|c}
\hline Video & $I_{s}$ & $P_{B}$ & \multicolumn{4}{|c}{ PSNR of different methods (dB) } \\
\cline { 4 - 7 } Sequence & & $(\%)$ & method 1 & method 2 & method 3 & method 4 \\
\hline Stefan & 3 & 10 & 33.2 & 31.9 & 33.6 & 34.9 \\
& 4 & 5 & 33.5 & 31.9 & 34.1 & 35.7 \\
\hline Football & 3 & 10 & 31.4 & 31.8 & 32.6 & 33.2 \\
& 4 & 5 & 31.4 & 31.8 & 33.0 & 33.4 \\
\hline Coastguard & 3 & 10 & 34.8 & 34.4 & 35.0 & 35.2 \\
& 4 & 5 & 34.7 & 34.4 & 35.1 & 35.3 \\
\hline Calendar & 3 & 10 & 33.9 & 34.3 & 34.3 & 35.6 \\
& 4 & 5 & 33.9 & 34.6 & 35.1 & 35.9 \\
\hline Mobile & 3 & 10 & 33.1 & 33.5 & 33.8 & 34.9 \\
& 4 & 5 & 33.7 & 33.6 & 33.8 & 34.9 \\
\hline Foreman & 3 & 10 & 34.2 & 35.8 & 35.9 & 36.1 \\
& 4 & 5 & 34.2 & 35.9 & 36.1 & 36.4 \\
\hline
\end{tabular}

To evaluate the performance of the joint flow control and JSCC approach, different simulations are conducted for the test sequence. These results allow us to observe the relative performance of the proposed error-resilience approach compared to the two fix-rate schemes where either no channel coding or the RS $(15,9)$ code is used exclusively. Furthermore, the proposed rate control scheme is used for all the above three approaches. In Fig. 6, we illustrate a plot of PSNR versus the frame number for the test sequence and the corresponding channel profile is illustrated in Table II. Obviously, the proposed JSCC scheme can be seen to achieve a much higher performance in terms of end-to-end PSNR compared to the no channel coding and fixed RS $(15,9)$ schemes. From the Fig. 6, we can see that although using the RS $(15,9)$ code can provide protection, a substantial performance loss is observed compared to the proposed JSCC scheme which is so flexible that it can achieve an optimal value according to current condition. In addition, although using no channel coding results in the best source coding efficiency, however, there are no error correcting capabilities which can be used to combat transmission errors. Therefore, the corresponding performance is considerably worse than the JSCC scheme.

In order to provide a more comprehensive evaluation of the proposed joint scheme, in Table III, we repeat the results for other QCIF video sequences under the same simulation configuration 
as the previous experiments (Note: method 1 is only JSCC without rate control, method 2 is only rate control without JSCC, method 3 is the joint flow control and fixed RS $(15,9)$ and method 4 is proposed joint flow control and JSCC). From Table III, it can be observed that the proposed joint rate control and JSCC scheme has considerable performance advantage comparing to the other competitive methods, which is due to the proposed scheme has the characteristics of network-adaptive and error-resilient. In addition, we also can observe that the imposed bit-plane $I_{s}$ and packet loss $P_{B}$ do play a important role. In particular, as the $I_{s}$ gets larger and the $P_{B}$ becomes smaller, the performance of the proposed scheme improves. It should be noted that all the simulation results in this table have been obtained using 300 runs in order to obtain statistically meaningful average values.

\section{CONCLUding Remarks}

In this paper, we use cross-layer technique to maximize the perceived video quality by combining distributed flow control with joint source-channel coding. At first, we propose a crosslayer distributed flow control scheme which can dynamically control the optimal transmission rate for wireless communications from source to destination. And then, we provide an analytical ratedistortion optimized joint source-channel coding approach to optimally select the channel coding strategy given the optimal transmission rate obtained from the proposed flow control scheme and the prevailing channel condition. Finally, the simulation results demonstrate the effectiveness of our proposed approach for multiple video streams over wireless multihop networks.

\section{ACKNOWLEDGMENTS}

This work is supported by the International Project PRA-SI (financed by France and China government) under Grant No. SI04-03, the Key Project of Nature Science Foundation of Jiangsu (China) under Grant BK2007729 and the Climbing Plan in NJUPT under Grant NY207061. Moreover, we also thank the anonymous reviewers for insightful comments and suggestions.

\section{APPENDIX I}

\section{Proof of Strictly Concave Function}

Taking derivatives again, for each of the nonlinear

$$
\sum_{i=1}^{n_{s}} \log \left[c_{l} p_{l} \prod_{k \in N_{I}(l)}\left(1-\sum_{m \in L_{o}(k)} p_{m}\right)-\sum_{n \in L_{I}\left(t_{l}\right)} c_{n} p_{n} \prod_{k \in N_{t o}^{I}(n), k \neq t_{l}}\left(1-\sum_{m \in L_{o}(k)} p_{m}\right)\right] \text { terms in } L_{P_{s}}\left(\tilde{P}_{s}\right),
$$


we obtain the Hessian

$$
H^{l}=\frac{-p_{l}}{\left(\sum_{k}\left(z_{l k}\right)+c_{l} p_{l}\right)^{2}}\left(\left(\sum_{k} z_{l k}+c_{l} p_{l}\right) \operatorname{diag}\left(z_{l}\right)-z_{l} z_{l}^{T}\right)
$$

where $z_{l k}=\exp \left(\tilde{p}_{l}\right)+c_{l} p_{l} \prod_{k \in N_{I}(l)}\left(1-\sum_{m \in L_{o}(k)} p_{m}\right)$, and $z_{l}$ is a column vector $\left[z_{l 1}, z_{l 2}, \ldots, z_{l L}\right]^{T}$. Matrix $H^{l}$ is indeed negative definite: for all vectors $v$,

$$
\begin{aligned}
v^{T} H^{l} v & =\frac{-p_{l}\left(\sum_{k}\left(z_{l k}\right)+c_{l} p_{l}\right)\left(\sum_{k \in N_{I}(l)}\left(1-\sum_{m \in L_{o}(k)} p_{m}\right) z_{l k}\right)}{\left(\sum_{k} z_{l k}+c_{l} p_{l}\right)^{2}} \\
& +\frac{p_{l}\left(\sum_{k \in N_{I}(l)}\left(1-\sum_{m \in L_{o}(k)} p_{m}\right) z_{l k}\right)^{2}}{\left(\sum_{k} z_{l k}+c_{l} p_{l}\right)^{2}}<0
\end{aligned}
$$

This is because the Cauchy Schwarz inequality $\left(a^{T} a\right)\left(b^{T} b\right) \geq\left(a^{T} b\right)^{2}$. Therefore, $L_{P_{s}}\left(\tilde{P}_{s}\right)$ is a strictly concave function of $\tilde{P}_{s}$, and its Hessian is a negative definite block diagonal matrix $\operatorname{diag}\left(H^{1}, H^{2}, \ldots, H^{L}\right)$.

\section{REFERENCES}

[1] M. G. Martini, M. Mazzotti et al., "Content adaptive network aware joint optimization of wireless video transmission”, IEEE Communications Magazine, vol.45, no.1, pp.84-90, 2007.

[2] W. Ge, J. Zhang, S. Shen, "A Cross-Layer Design Approach to Multicast in Wireless Networks", IEEE Transactions on Wireless Communications, vol.6, no.3, pp.1063- 1071, 2007.

[3] W.H. Wang, M. Palaniswami, and S. H. Low, "Optimal Flow Control and Routing in Multi-Path Networks", Performance Evaluation, vol.52, no.2-3, pp.119-132, 2003.

[4] S. Cho, W. A. Pearlman, "A full-featured, error-resilient, scalable wavelet video codec based on the set partitioning in hierarchical trees (SPIHT) algorithm”, IEEE Transactions on Circuits and Systems for Video Technology, vol.12, no. 3, pp.157-171, 2002.

[5] J. Kim, R. M. Mersereau, Y. Altunbasak, "Distrubuted Video Streaming Using Multiple Description Coding and Unequal Error Protection”, IEEE Transactions on Image Processing, vol.14, no.7, pp. 849-861, 2005.

[6] R. Tian, Q. Zhang, et al., "Robust and efficient path diversity in application-layer multicast for video streaming", IEEE Transactions on Circuits and Systems for Video Technology,vol.15, no.8, pp.961-972, 2005.

[7] G. Wang, S. Futemma, E. Itakura, "Multiple-Description Coding for Overlay Network Streaming”, IEEE Multimedia, vol.14, no.1, pp.74-82, 2007.

[8] C.-M. Fu, W.-L. Hwang, et al., "A Joint Source and Channel Coding Algorithm for Error Resilient SPIHT-Coded Video Bitstreams”, Journal of Visual Communication \& Image Representation, vol.17, pp. 1164-1177, 2006.

[9] X. Lin, Ness B. Shroff, "Utility Maximization for Communication Networks with Multi-path Routing”, IEEE Trans. on Automatic Control, vol.51, no.5, pp.766-781, 2006. 
[10] L. Chen, Steven H. Low, Mung Chiang, and John C.Doyle, "Cross-layer Congestion Control, Routing and Scheduling Design in Ad Hoc Wireless Networks", INFOCOM, pp. 321-334, 2006.

[11] R. T. Rockafellar, "Monotone Operators and the Proximal Point Algorithm", SIAM J. Control and Optimization, vol. 14, pp. 877-898, Aug. 1976.

[12] L. Zhou, B. Zheng, B. Geller, A. Wei, S.Xu, and Y. Li, "Cross-layer rate control, medium access control and routing design in cooperative VANET”, Computer Communication (2008), doi: 10.1016/j.comcom.2007.12.006

[13] L. Xiao. M. Johnasson and S. Boyd, "Simultaneous routing and resource allocation via dual decomposition", IEEE Trans. on Communications, vol.52, no.7, pp. 1136-1144, July 2004.

[14] S. H. Low, "A duality model of TCP and queue management algorithms", IEEE/ACM Trans. on Networking, vol. 11, no. 4, pp. 525-536, Aug. 2003.

[15] Q. Qu, Y. Pei, J. W. Modestino, X. Tian, and B. Wang “Cross-layer QoS control for video communications over wireless ad hoc networks", EURASIP Journal on Wireless Communications and Networking, vol.5, no.5, pp. 743-756, Oct. 2005.

[16] X. Zhu, E. Setton, and B. Girod, "Congestion-distortion optimized video transmission over ad hoc networks", Signal Processing: Image Communication, vol. 20, no. 8, pp. 773-783, Sep. 2005. 